\title{
Development of New Predictive Equations to Estimate Basal Metabolic Rates in Iranian Adults: A Study Protocol
}

\author{
Bahareh Nikooyeh $^{1}$, Tirang R. Neyestani ${ }^{1 *}$ \\ 1- Laboratory of Nutrition Research, National Nutrition and Food Technology Research Institute and Faculty of Nutrition Sciences and Food \\ Technology, Shahid Beheshti University of Medical Sciences, Tehran, Iran.
}

\section{A B S T R A C T}

Background and Objectives: Studies indicate over-estimation of basal metabolic rate (BMR) using common equations for the Asian people. The present study aims to develop new predictive equations for the Iranian people and to compare these equations with commonly used formulas.

Materials and Methods: Total, 150healthy subjects aged 18-60 yrare invited to the Laboratory of Nutrition Research, National Nutrition and Food Technology Research Institute. Demographic data are gathered using a questionnaire. Then, anthropometric measures are taken and blood sampling is done for thyroid function tests. If the subject merits all the inclusion criteria, indirect calorimetry will be performed. The value of BMR will be predicted using common equations (Harris-Benedict, FAO/WHO/UNU, Miffilin).

Differences between predicted (using equations) and measured (using indirect calorimetry) values are estimated. Correlations between the two sets of data is performed using Pearson or Spearman coefficients. Between-method agreement is checked using Bland-AltmanPlot. Accuracy of the predicted values using equations isconsidered as the proportion of participants whose calculated BMR is $90-110 \%$ of their measured BMR. Multiple regression analysis is employed to develop new predictive equations for the BMR based on the independent variables.

Conclusions: Since facilities for the measurement of BMR may not be accessible in many clinical or research settings, BMR is usually estimated using predictive equations. However, several studies have reported inaccuracy of these equations for certain populations. Therefore, development of new population-specific predictive equations seems reasonable. These equations could hopefully reduce the energy estimation errors both in clinical nutritional interventions and community-based nutrition researches.

Keywords: Basal metabolic rate, Indirect calorimetry, Equations, Iran

\section{Introduction}

Basal metabolic rate (BMR), the minimum energy required for survival, comprises nearly $60 \%$ of the total energy requirement in a person with usual physical activity. BMR depends mostly on body composition (fat and fat-free masses) as well as gender, age and race (1). To determine BMR, direct and indirect calorimetrymay be used. The direct method needs sophisticated facilities and trained staff and is therefore used mostly in specialized research centers. The indirect method is commonly based on analysis of the inspired oxygen and expired carbon dioxide.Though indirect method is far less expensive and more feasible than direct method, its application is actually limited to some clinics and hospitals. Instead, in many clinical settings as well as epidemiologic studies, BMR is estimated using various equations (1).

The predictive equations are usually based on the age, gender, height and weight of the participants. Harris-Benedict (2), Schofield (3), FAO/WHO/ UNU (4) and Mifflin (5) are the most commonly used formulas. Some studies have endorsed Mifflin equation as a more accurate estimation, compared to other equations (6) whereas a population-based study found Harris-Benedict more practical in Iranian community (7). In contrast, findings from several 
studies revealed over-estimation of BMR using these equations for the Asian people (8-11). It has been suggested that BMR in Asian people could be 15-20\% less than in European and American people (12-13). One of the factors affecting inter-racial differences in BMR includes differences in body compositions, notably percent of fat mass (FM) and lean body mass (LBM). Asian people, compared with their Caucasian counterparts, have more body fat and hence less LBM (14).

This study aims to develop new predictive equations for the Iranian community and to compare them with commonly used equations namely Harris-Benedict, FAO/WHO/UNU and Mifflin-St. Jeor.

\section{Subject and Methods}

\section{Calculation of sample size}

Sample size can be calculated based on the objectives of the study.

\section{Evaluation of between-method agreement}

Considering the standard deviation (SD) of differences between indirect calorimetry and HarrisBenedict equation in the previous studies (15) which was $244 \mathrm{kcal}$ and using Bland-Altman for evaluation of method agreement (16), a total of 150 subjects could give a confidence interval of $\pm 68 \mathrm{kcal}$ for upper and lower limits of the agreement range.

\section{Development of regression models}

According to the previous studies (17), with three independent variables and $\mathrm{R} 2=$ (at least) 0.5 , a total of 130 subjects will be needed.

Altogether, at most 150 subjects will be needed.

\section{Study protocol}

Healthy adult subjects aged 18-60 years of old are invited to the Laboratory of Nutrition Research, National Nutrition and Food Technology Research Institute, Tehran, Iran. A comprehensive explanation of the study protocol and its objectives is given to the participants before they sign an informed written consent. Demographic data are gathered using questionnaires and face-to-face interviews. Then, anthropometric measures are taken and blood sampling is done for thyroid function tests. If the subject merits all the inclusion criteria, indirect calorimetry will be performed.

\section{Subjects}

Apparently healthy adults who meet the following inclusion criteria:

(a) Aged 18-90 yrs (b) Normal thyroid function

(c) Not pregnant, lactating or menses at the time of assessments

(d) Not having clinical diseases including endocrine, cardiac, renal, hepatic and malignant diseases

(e) Not using medications affecting BMR including drugs used for cardiac and endocrine disorders as well chemotherapeutic agents

(f) The subject should not be a professional athlete

\section{Assessments}

(a) Demographic data

Demographic data are collected by a trained nutritionist using face-to-face interviews and questionnaires.

(b) Anthropometric measurements.

Weight is measured with light clothing and without shoes using a digital scale (Seca 808; Seca, Hamburg, Germany) to the nearest of $0.1 \mathrm{~kg}$. Height is measured using a stadiometer (Seca 216, Seca, Hamburg, Germany) to the nearest of $0.1 \mathrm{~cm}$. Body mass index (BMI) is calculated using the equation body weight $(\mathrm{kg}) /$ height $^{2}(\mathrm{~m})$. Categorization of the participants based on BMI is as: underweight: $<18.4 \mathrm{~kg} / \mathrm{m}^{2}$; normal weight: $18.5-24.9 \mathrm{~kg} / \mathrm{m}^{2}$; overweight: 25.0 $29.9 \mathrm{~kg} / \mathrm{m}^{2} ;$ and obese > $30.0 \mathrm{~kg} / \mathrm{m}^{2}$. Waist circumference (WC) and hip circumference (HC) and were both measured using a measuring tape to the nearest $0.1 \mathrm{~cm}$.

(c) Body composition analysis

Total body compositionanalysis is done using bioelectrical impedance analysis (BIA) system (Quadscan 4000, Bodystat, Isle of Man, UK) whereas truncal and visceral fat analysis is performed by applyingViscan(AB140, Tanita, Tokyo, Japan).

(d) Laboratory investigations

Blood sampling and handling. After an overnight fasting, $10 \mathrm{~mL}$ venous blood is taken from each participant. Blood samples kept for $30-45 \mathrm{~min}$ at room temperature (RT) are centrifuged at 800 gat RT for $30 \mathrm{~min}$. Sera are recovered, aliquoted in fresh microtubes and kept at $-80^{\circ} \mathrm{C}$ until the day of analysis.

Thyroid function tests. Serum concentrations of thyroid stimulating hormone (TSH), free thyroxin (fT4; both from PishtazTeb, Tehran, Iran) and antithyroid peroxidase antibody (anti-TPO-Ab; Aeskulisa, Wendelsheim, Germany) are determined using enzyme immunoassay (EIA) commercial kits. The pre-defined normal ranges are as following:

TSH: 0.32-5.2 mIU/L 
Anti-TPO-Ab: Normal < $40 \mathrm{IU} / \mathrm{mL}$, Equivocal 40-60 IU/mL, Positive $>60 \mathrm{IU} / \mathrm{mL}$

fT4: $0.7-1.8 \mathrm{ng} / \mathrm{dL}$

In this study, participants with high levels of antiTPO (up to $500 \mathrm{IU} / \mathrm{mL}$ ) but normal TSH and fT4 are considered as euthyroid. However, participants with deranged TSH and/or fT4 with or without concomitant above normal range anti-TPO levels are excluded(18-19).

(e) Indirect calorimetry

Indirect calorimetry is performed using respiratory gas analyzer (Fitmate pro, Cosmed, Rome, Italy) at RT while the subject is $12 \mathrm{hr}$ fasting and lies down.

\section{Statistical analyses}

Mean and standard deviation (SD) are used to describe quantitative data while absolute and relative frequencies were applied for qualitative variables. Normality of data distribution is evaluated using Shapiro-Wilk test. Differences between the predicted (using equations) and measured (using indirect calorimetry) values are estimated. Evaluation of correlation between the two sets of data is performed by Pearson ( $r$; for data with normal distribution) or Spearman $\left(\mathrm{r}_{\mathrm{s}}\right.$; for data with non-normal distribution) equations. Between-method agreement is checked by Bland-Altman. Percent of bias of all equations compared to measured value will be calculated. Accuracy of predicted values using equations is considered as the proportion of the subjects whose calculated BMR is $90-110 \%$ of their measured BMR. Multiple regression analysis is employed to develop new predictive equations for BMR based on independent variables (age, gender, weight and height). In this study $\mathrm{p}<0.05$ is considered as statistically significant. All statistical analyses are performed by using Statistical Package for Social Science (SPSS version 21; SPSS Inc, Chicago, IL).

\section{Discussion}

Successful nutritional interventions involve accurate prediction of energy requirement. As the facilities to measure BMR may not be accessible or applicable in many clinical or research settings, BMR is usually estimated using predictive equations. However, several studies have reported inaccuracy of these equations for certain populations $(1,13,20-22)$. Body composition and more specifically percent of FM and LBM are among the determinants of RMR (23-24) and body composition may differ in various ethnicities (25). Development of new populationspecific predictive equations, therefore, seems plausible. These equations could hopefully reduce the energy estimation errors both in clinical nutritional intervention and community-based nutrition research.

\section{Financial disclosure}

The authors declare no conflict of interests.

\section{References}

1. Pavlidou E, Petridis D, Tolia M, Tsoukalas N, Poultsidi A, Fasoulas A, et al. Estimating the agreement between the metabolic rate calculated from prediction equations and from a portable indirect calorimetry device: an effort to develop a new equation for predicting resting metabolic rate. Nutr Metab (Lond). 2018;15:41.

2. Roza AM, Shizgal HM. The Harris Benedict equation reevaluated: resting energy requirements and the body cell mass. The American journal of clinical nutrition. 1984;40(1):168-82.

3. Schofield W. Predicting basal metabolic rate, new standards and review of previous work. Human nutrition Clinical nutrition. 1985;39:5-41.

4. Food, Nations AOotU. Energy and Protein Requirements: Report of a Joint FAO/WHO/UNU Expert Consultation: WHO.; 1985.

5. Mifflin MD, St Jeor ST, Hill LA, Scott BJ, Daugherty SA, Koh YO. A new predictive equation for resting energy expenditure in healthy individuals. The American journal of clinical nutrition. 1990;51(2):2417.

6. Frankenfield DC. Bias and accuracy of resting metabolic rate equations in non-obese and obese adults. Clinical nutrition. 2013;32(6):976-82.

7. Amirkalali B, Hosseini S, Heshmat R, Larijani B. Comparison of Harris Benedict and Mifflin-ST Jeor equations with indirect calorimetry in evaluating resting energy expenditure. Indian journal of medical sciences. 2008;62(7):283.

8. Case KO, Brahler CJ, Heiss C. Resting energy expenditures in Asian women measured by indirect calorimetry are lower than expenditures calculated from prediction equations. Journal of the American Dietetic Association. 1997;97(11):1288-92.

9. Leung R, Woo J, Chan D, Tang N. Validation of prediction equations for basal metabolic rate in Chinese subjects. European journal of clinical nutrition. 2000;54(7):551.

10. Azcue M, Pencharz P, Zello G. Are the FAO/WHO/UNU predictive equations for resting metabolic rate accurate? The American journal of clinical nutrition. 1991;54(3):613-4.

11. Nhung B, Khan N, Hop L, Lien D, Le D, Hien V, et al. $\mathrm{FAO} / \mathrm{WHO} / \mathrm{UNU}$ equations overestimate resting 
metabolic rate in Vietnamese adults. European journal of clinical nutrition. 2005;59(10):1099.

12. Tseng C-K, Hsu H-S, Ho C-T, Huang H-Y, Liu C-S, Lin $\mathrm{C}-\mathrm{C}$, et al. Predictive equation of resting energy expenditure in obese adult Taiwanese. Obesity research \& clinical practice. 2011;5(4):e313-e9.

13. Rao Z-y, Wu X-t, Liang B-m, Wang M-y, Hu W. Comparison of five equations for estimating resting energy expenditure in Chinese young, normal weight healthy adults. European journal of medical research. 2012;17(1):26.

14. Who EC. Appropriate body-mass index for Asian populations and its implications for policy and intervention strategies. Lancet (London, England). 2004;363(9403):157.

15. Shaneshin M, Rezazadeh A, Jessri M, Neyestani T, Rashidkhani B. Validity of predictive equations for resting energy expenditure among Iranian women. Asia Pac J Clin Nutr. 2011;20(4):646-53.

16. Bland JM, Altman DG. Statistical methods for assessing agreement between two methods of clinical measurement. International Journal of Nursing Studies. 2010;47(8):931-6.

17. Knofczynski GT, Mundfrom D. Sample sizes when using multiple linear regression for prediction. Educational and psychological measurement. 2008;68(3):431-42.

18. Hutfless S, Matos P, Talor MV, Caturegli P, Rose NR. Significance of prediagnostic thyroid antibodies in women with autoimmune thyroid disease. The Journal of Clinical Endocrinology \& Metabolism. 2011;96(9):E1466-E71.
19. Tipu HN, Ahmed D, Bashir MM, Asif N. Significance of Testing Anti-Thyroid Autoantibodies in Patients with Deranged Thyroid Profile. Journal of thyroid research. 2018;2018.

20. Miller S, Milliron BJ, Woolf K. Common Prediction Equations Overestimate Measured Resting Metabolic Rate in Young Hispanic Women. Top Clin Nutr. 2013 Apr;28(2):120-35.

21. Nhung BT, Khan NC, Hop LT, Lien DT, Le DS, Hien $\mathrm{VT}$, et al. FAO/WHO/UNU equations overestimate resting metabolic rate in Vietnamese adults. Eur J Clin Nutr. 2005 Oct;59(10):1099-104.

22. Song T, Venkataraman K, Gluckman P, Seng CY, Meng KC, Khoo EY, et al. Validation of prediction equations for resting energy expenditure in Singaporean Chinese men. Obes Res Clin Pract. 2014 MayJun;8(3):e201-98.

23. McMurray RG, Soares J, Caspersen CJ, McCurdy T. Examining variations of resting metabolic rate of adults: a public health perspective. Medicine and science in sports and exercise. 2014;46(7):1352.

24. Woods AL, Rice AJ, Garvican-Lewis LA, Wallett AM, Lundy B, Rogers MA, et al. The effects of intensified training on resting metabolic rate (RMR), body composition and performance in trained cyclists. PLoS One. 2018;13(2):e0191644.

25. Heymsfield S, Peterson C, Thomas D, Heo M, Schuna Jr J. Why are there race/ethnic differences in adult body mass index-adiposity relationships? A quantitative critical review. Obesity reviews. 2016;17(3):262-75. 